CÓMO CITAR ESTE ARTÍCULO:

Cárdenas, L. F.; Espinosa, N.; González, O. V. y Guasca, H. C. (2016). Influencia de la tecnificación panelera en los medios de vida de productores rurales en la vereda Junco, San Benito (Santander). Revista de Sociología y Antropología: VIRAJES, 18 (2), 115-136. DOI: 10.17151/rasv.2016.18.2.8

\title{
ryin \\ INFLUENCIA DE LA TECNIFICACIÓN PANELERA EN LOS MEDIOS DE VIDA DE PRODUCTORES RURALES EN LA VEREDA JUNCO, SAN BENITO (SANTANDER)*
}

\author{
LUISA FERNANDA CÁRDENAS FRANCO** \\ NATALIA ESPINOSA BECERRA*** \\ OLGA VIVIANA GONZÁLEZ PULIDO**** \\ HIDALI CARINA GUASCA PARRA*****
}

Recibido: 30 de Marzo de 2016

Aprobado: 20 de Diciembre de 2016

Artículo de Investigación

\footnotetext{
* A Artículo de Investigación INFLUENCIA DE LA TECNIFICACIÓN PANELERA EN LOS MEDIOS DE VIDA DEL PRODUCTOR RURAL EN LA VEREDA JUNCO MUNICIPIO DE SAN BENITO (SANTANDER) Febrero 2015 - Mayo 2016 Fundación Universitaria Juan de Castellanos (Tunja, Boyacá) ** H Estudiante de X Semestre de Trabajo Social. Miembro del Semillero de Investigación Newén Mapu, Grupo de Investigación DOTEZ. Fundación Universitaria Juan de Castellanos. Tunja, Boyacá-Colombia. E-mail: luisafer1908@hotmail.com (ㅇ) ORCID: 0000-0002-0616-2102

*** M.Sc (c) en Desarrollo Rural, Pontificia Universidad Javeriana, Socióloga, Universidad Nacional de Colombia. Docente Investigadora, Grupo de Investigación DOTEZ. Fundación Universitaria Juan de Castellanos. Tunja Boyacá-Colombia. E-mail: nespinosa@jdc.edu.co @ ORCID: 0000-0002-4881-1262

**** Estudiante de X Semestre de Trabajo Social. Miembro del Semillero de Investigación Newén Mapu, Grupo de Investigación DOTEZ.Fundación Universitaria Juan de Castellanos. Tunja, Boyacá-Colombia. E-mail: vivianag234@gmail.com @ ORCID: 0000-0003-3855-4060

***** Estudiante de X Semestre de Trabajo Social. Miembro del Semillero de Investigación Newén Mapu, Grupo de Investigación DOTEZ. Fundación Universitaria Juan de Castellanos. Tunja, Boyacá-Colombia. E-mail: hidalicarinag@gmail.com (이 ORCID: 0000-0003-3810-545X
} 


\title{
Resumen
}

Este artículo muestra el resultado de una investigación realizada en la Vereda Junco, San Benito (Santander-Colombia) sobre la influencia de la tecnificación panelera en los Medios de Vida de productores rurales a partir de la identificación, análisis y correlación de los elementos que constituyen los capitales Humano, Social, Financiero, Físico y Natural como formas de subsistencia de dicha población. Esta investigación cualitativa es descriptiva, con un diseño narrativo y sustentada teórica y metodológicamente bajo el Enfoque de los Medios de Vida Sostenibles (MVS) empleando como elemento central el Pentágono de Bienestar como punto de referencia en la ilustración de la información. Los resultados evidencian los cambios que han tenido los capitales con relación a la actividad panelera en términos de conocimientos, prácticas productivas, relaciones sociales, medio ambiente, infraestructura física y aspectos económicos, lo cual permite entender de manera holística la situación actual de los productores paneleros.

Palabras clave: Medios de Vida Sostenibles, tecnificación panelera, productor rural, capitales, economía campesina.

\section{TECHNIFICATION PANELA'S INFLUENCE OF THE LIVELIHOOD'S PRODUCERS RURAL LIFE IN THE VEREDA JUNCO, SAN BENITO (SANTANDER)}

\begin{abstract}
This paper shows an investigation's results in the Vereda Junco, San Benito (Santander-Colombia) about the influence of technicalization of panela in the Livelihoods of rural producers from the identification, analysis and correlation of the elements of capitals: human, social, financial, physical and natural with which this population has to survive. This qualitative research is descriptive, with a narrative design and is supported theoretical and methodological approach under the Sustainable Livelihoods, using as central element the Pentagon of Well-being like point of reference in the graphical illustration of the information. The results shows the changes that have taken capital in relation to the panelera activity in terms of knowledge, production practices, social relations, environment, physical infrastructure and economic aspects, which allow us to understand holistically the current situation of panela's producers.
\end{abstract}

Key words: Sustainable livehoods, panela's technicalization, rural producer, rural development, peasant economy. 


\section{Introducción}

olombia es el segundo productor mundial de panela después de la India, y a nivel nacional el cultivo de la caña (Saccharum officinarum) ocupa dentro de los cultivos permanentes el segundo lugar en extensión después del café con 249.384 hectáreas (Osorio, 2007). La agroindustria de la panela en Colombia se desarrolla principalmente en la región Andina como una estrategia de generación de ingresos para más de 70.000 familias campesinas, que responden a una significativa demanda nacional y a un consumo arraigado en los sectores urbanos y rurales del país (García, 2015).

La producción de panela se ha posicionado como una de las actividades económicas más importantes en la economía nacional, y ha experimentado una serie de cambios en las formas de producción con el objetivo de mejorar la calidad de la panela y reducir el tiempo del proceso productivo, y así poder responder a las demandas del mercado globalizado. En el siglo XXI, los productores paneleros se han visto en la necesidad de modernizar sus trapiches, debido a los avances tecnológicos y la normatividad que los regula.

Lo anterior ha generado una serie de cambios en los Medios de Vida de las comunidades rurales, principalmente en los recursos a los cuales tienen acceso, según el Department for International Development (DFID, 1999) se enmarcan dentro del concepto capital, y se clasifican en los siguientes:

El capital natural se refiere a los recursos naturales que cuenta cada ser humano para subsistir: el agua, la tierra. El capital humano encierra elementos relacionados con las capacidades, habilidades, aptitudes y conocimientos, que se relevan generacionalmente. El capital social, corresponde a las relaciones sociales que se originan en lo familiar, grupal y comunitario, y desde la organización gremial, productora y política. El capital financiero contempla los recursos económicos que tienen las personas para poder satisfacer sus necesidades. Finalmente, el capital físico, se refiere a la infraestructura básica y productiva (Gottret, 2011). Es importante mencionar que éste último capital experimenta una serie de cambios por la tecnificación agrícola.

Según la Superintendencia de Industria y Comercio de Colombia, dentro de los departamentos catalogados como mayores productores de panela en el país se encuentran: Cundinamarca, Santander, Antioquia, Boyacá y Nariño, departamentos que concentran más de las dos terceras partes de la producción nacional (SIC, 2012, p.7). El sector del país que ha alcanzado mayores rendimientos y ha conseguido un grado de desarrollo tecnológico tanto en el cultivo de caña panelera como en su procesamiento 
(Rodríguez, García, Roa y Santacoloma, 2004) es la Hoya del Río Suarez, conformada por los departamentos de Boyacá y Santander (SIC, 2012), lo cual ha influido notablemente en la capacidad de producción y la competitividad de la región.

Entre los municipios que conforman la Hoya del Río Suarez, se encuentra San Benito, Santander (Alcaldía Municipal de San Benito, 2012, p. 60) uno de los principales productores de panela, debido a que casi el $70 \%$ de su territorio está dedicado a la producción de caña panelera. En la región predomina la explotación de pequeña o mediana escala, donde se evidencia una integración al mercado de tipo comercial tanto en materia de generación de empleo (Rodríguez, 1998, p.37) y compra de insumos, como en la venta de la panela, pero persisten aún características de economía tradicional como la presencia de la aparcería: en este tipo de producción los empleados son contratados por temporadas para trabajar únicamente durante las moliendas y la mayoría de veces, se les paga conforme a la panela producida, lo cual genera una inestabilidad laboral y repercute de una $\mathrm{u}$ otra manera en la calidad de vida de los trabajadores.

El estudio está orientado a describir la influencia de la tecnificación panelera en los capitales humano, financiero, natural, social y físico del productor rural de la vereda Junco en el municipio de San Benito (Santander) a partir del Enfoque de los Medios de Vida.

\section{Metodología}

La investigación es de tipo descriptiva, puesto que está orientada a realizar una descripción detallada de la influencia de un fenómeno, en este caso, de la tecnificación panelera, en los capitales que conforman los Medios de Vida de una población rural (Danhke, 1986, citado en Hernández Sampieri, Fernández y Baptista, 2010, p. 117), es decir, este tipo de investigación permitió comprender el fenómeno a estudiar a partir de la caracterización del mismo, teniendo en cuenta la experiencia del productor panelero desde en el contexto temporal y geográfico en el que se desenvuelve (Rada, 2007). Además, cabe resaltar que dicha descripción se realizó con base en una serie de categorías (García, 2004), contempladas dentro de los objetivos de la investigación, las cuales se mencionarán más adelante.

La metodología que se implementó para llevar a cabo la investigación se articuló mediante un diseño narrativo, en el que según Hernández Sampieri et al. (2010) el investigador recolecta datos sobre las experiencias de ciertas personas para describirlas y analizarlas, y se sugiere "utilizar 
una perspectiva que provee de estructura para entender al individuo o grupo y escribir la narrativa (se contextualiza la época y el lugar donde vivió la persona o grupo)" (p. 506) con el fin de reconstruir las vivencias de las personas, casi siempre de manera cronológica. Para este estudio se obtuvieron las narrativas a partir de entrevistas semi-estructuradas, en los distintos acercamientos a los productores paneleros de la Vereda Junco, y se realizó el análisis por las categorías establecidas en la investigación.

La investigación estuvo orientada hacia el tipo de muestreo no probabilístico, ya que se caracteriza porque no se conoce la probabilidad de que una unidad de la población sea incluido en la muestra, y se basa en un juicio subjetivo (Salamanca, 2008), en la investigación realizada en la Vereda Junco, las investigadoras en el primer acercamiento con la comunidad no estimaron una muestra representativa, el número de participantes en el estudio fue establecido, en función de obtener información necesaria a partir de los relatos de los pobladores, al cual respondiera a los objetivos.

La muestra perteneciente a la investigación, estuvo seleccionada bajo los criterios cualitativos de muestra en cadena o por redes "bola de nieve" y por conveniencia. Según Bisquerra (2009) en el muestreo de bola de nieve "Se localizan algunos individuos, los cuales conducen a otros, y estos a otros, y así hasta conseguir una muestra suficiente" (p.149) en la investigación realizada se estableció contacto con un participante clave, habitante de la vereda Junco, que permitió a las investigadoras una aproximación a los productores paneleros.

Por su parte Hernández Sampieri et al. (2010) afirman que el tipo de muestra por conveniencia se refiere a "simplemente casos disponibles a los cuales tenemos acceso" (p.401), este tipo de muestra caracteriza por ser un proceso sencillo y económico que permite acceder a otros tipos de muestras durante el desarrollo de la investigación (Crespo y Salamanca, 2007) la investigación fue realizada en la vereda Junco porque el participante clave tenía una mayor facilidad de realizar contacto con los productores paneleros, quienes podían proporcionar información acerca del fenómeno de la tecnificación panelera, y esto contribuía a tener un mejor acceso a la población reunida en un mismo sector del municipio. La muestra seleccionada de informantes corresponde a 16 productores paneleros; con modalidad de cañicultor y dueños de trapiche, la población está dividida en 3 mujeres y 13 hombres pertenecientes a la vereda Junco.

Se establecieron tres fases que permitieron desarrollar de una manera continua el proceso de investigación: a. Revisión documental, b. Recolección de Información y c. Análisis de la Información. 


\section{a) Revisión documental}

Para la primera fase se llevó a cabo una revisión documental para comprender el Enfoque de los Medios de Vida, el proceso de tecnificación y producción panelera, elementos generales sobre el sector rural colombiano y de la región, e información general sobre el municipio para poder contextualizar el ejercicio investigativo y con esto la elección de la metodología adecuada para la recolección de información acorde al tipo de población con el que se trabajó.

\section{b) Recolección de Información}

Como técnica central en la recolección de información se encuentra la elaboración de entrevistas semi-estructuradas entendida "como una guía de preguntas, en donde el entrevistador tiene la libertad de introducir preguntas adicionales para precisar conceptos u obtener mayor información sobre los temas deseados, es decir, no todas las preguntas están predeterminadas" (Grinnnell y Unrau, 2007, citados en Hernández Sampieri et al, p. 418). Para la creación de las guías de preguntas se elementos generales de las categorías de análisis.

Usar este tipo de técnica permitió obtener respuestas sobre el tema y problema tópico de interés, en los términos de lenguaje y la perspectiva del entrevistado, que en este caso correspondió a productores rurales, lo importante incurrió sobre el contenido y la narrativa de cada respuesta de los participantes (Hernández Sampieri et al, 2010).

Para la ejecución de esta fase, se tuvo en cuenta las categorías de análisis desarrolladas por objetivo y relacionadas por etapas (Etapa 1: Historia de la tecnificación panelera -Etapa 2: Reconociendo los capitales), teniendo en cuenta que la técnica transversal al proceso es la entrevista semi-estructurada, se tuvo además como apoyo diversas herramientas que permitieron recolectar la información de una manera más dinámica, completa y con mayor empatía por parte del entrevistado.

\section{Etapa 1. (Historia de la Tecnificación panelera)}

Cumple con el primer objetivo específico, frente a los rasgos y/o características sobre su historia, mediante la realización una entrevista semi- estructurada para obtener información sobre dicho proceso; como herramienta de apoyo se empleó una línea de tiempo, que tiene como objetivo según Geilfus (2002) "saber cuáles han sido los cambios significativos en el pasado de la comunidad, los cuales tienen su influencia en los eventos y actitudes del presente" (p.53). De este modo, se pudo 
identificar las categorías de análisis de una forma clara y congruente que permitieron relacionar la información obtenida.

\section{Etapa 2. (Reconociendo los capitales)}

Teniendo en cuenta, la principal técnica empleada(la entrevista semiestructurada) y las herramientas de apoyo adaptadas según las necesidades de cada uno de los capitales, se utilizó para la recolección de información relacionada con el capital social, el Diagrama de Venn (Organización Social) que para Geilfus (2002) "tiene como principal objetivo aprender sobre las organizaciones y grupos activos en la comunidad, y como sus miembros los visualizan; entender las interacciones que tienen estas organizaciones entre sí" (p.41), para su realización se debió incluir personas representativas de los diferentes sectores presentes en la comunidad, y por necesidades de la investigación este componente se adaptó y se trabajó de manera individual en el proceso de entrevista. La utilidad del ejercicio radicó en la comparación de las visiones de diferentes actores.

Para el capital natural y físico, se realizó un mapeo de finca antes y después de la tecnificación panelera, que según Geilfus (2002), puede hacerse a nivel grupal, o de manera individual en la que cada participante (o unos escogidos) elabora el mapa de su finca con la ayuda de los demás. En el caso de trabajo grupal, se va a privilegiar el aspecto educativo pero tal vez limitar el nivel de detalle; a nivel familiar se puede hacer participar a todo el núcleo familiar y llevar a mayor detalle. Hay que tratar que la mayoría de miembros de la familia participen para limitar sesgos de género o edad. Para este estudio debido a la dificultad de congregar de manera grupal a todos los participantes de la investigación se debió hacer el mapeo en cada finca.

Además se realizó una entrevista base para la identificación de elementos importantes dentro del capital natural, capital físico, capital humano y capital financiero con guías de preguntas específicas.

Como herramienta de apoyo, para la retroalimentación de cada uno de los capitales, fue usado el gráfico histórico de la comunidad, orientado a la comprensión de las tendencias de los capitales, a partir del auge de la tecnificación panelera, el cual permitió hacer una matriz en la que se mostraron los principales cambios que han experimentado los productores en diversos aspectos a través de los diferentes espacios del tiempo (Geilfus, 2002). 


\section{c) Análisis de la información}

Para analizar la información, se tuvo en cuenta el diseño investigativo donde se muestran las transcripciones de las narrativas obtenidas durante el proceso, las cuales fueron clasificadas, a través de un proceso de marcación por colores, teniendo en cuenta, que se identificaron elementos de los diferentes capitales en cada uno de los resultados obtenidos tras la aplicación de las herramientas, esto debido a la conexión entre cada uno de ellos.

Como segundo paso la información obtenida en la marcación se ubicó en una matriz que permitió consolidar los registros por categorías de análisis y por entrevistados, posteriormente se tomó un apartado de la información de manera textual, se hizo un comentario por parte de las investigadoras que dio paso a comprender los resultados, y de este modo hacer la construcción del análisis con base en la revisión documental consolidando así los capítulos: "Reconstruyendo la historia de la producción panelera en San Benito" y "La dinámica entre los capitales de los productores".

Posteriormente se explicó la dinámica entre los capitales, desde el Enfoque de Medios de Vida, mediante la elaboración del "pentágono de bienestar", donde se encuentran los capitales en su nivel ideal, de manera paralela se construyeron: el primero que correspondió a los Medios de Vida previos al auge de la tecnificación y el segundo a los actuales; cabe aclarar que el ejercicio no se realizó con el fin de calificar a la población, si no por el contrario, de mostrar gráficamente la interpretación de los datos obtenidos, procurando hacer un ejercicio objetivo.

Para la ponderación del "pentágono de bienestar" se especificó una escala de 1 a 10, donde el 1 corresponde en menor presencia del capital y el 10 a la mayor presencia del mismo. Dicha ponderación fue justificada y respaldada en la información obtenida, con relación a la descripción de los capitales.

El pentágono de la situación o estado ideal, es la representación gráfica de la cúspide del bienestar, en términos del acceso y manejo de los activos con los que cuenta una comunidad para subsistir. En la figura 1, se muestra la máxima puntuación que puede obtener un capital, lo cual se refleja en el perímetro externo, mientras que el punto central en donde se unen los ejes, corresponde a un mínimo acceso. 


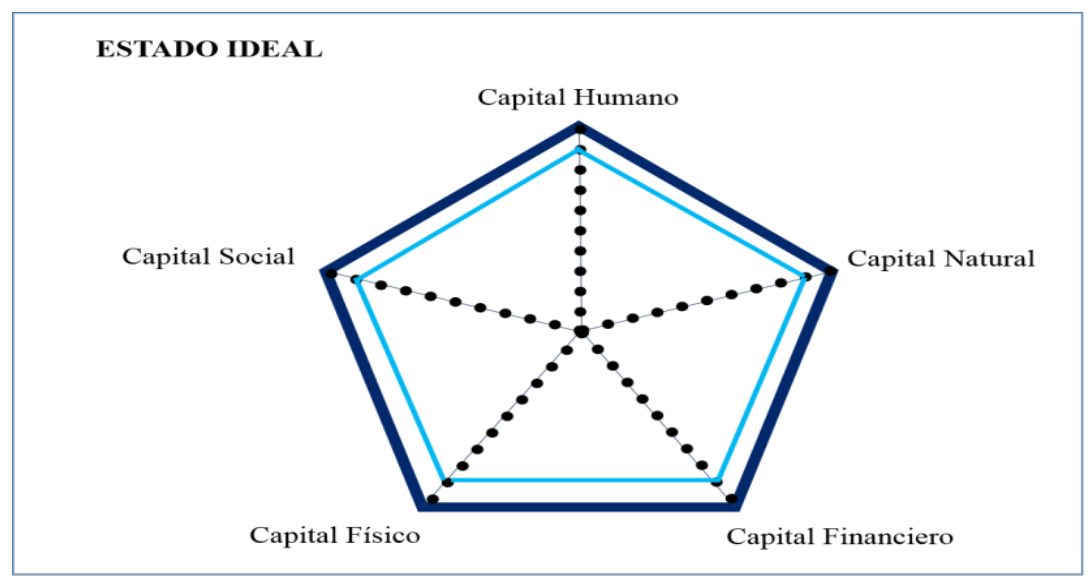

Figura 1. Pentágono de estado ideal

Fuente: DFID, 1999

Para terminar, se hizo un análisis entre los dos pentágonos de bienestar elaborados, procurando en lo posible establecer la influencia de la tecnificación panelera en los Medios de Vida del productor en la vereda Junco, por medio de las gráficas, de la información recopilada y de la revisión de literatura.

\section{Resultados y discusión: tecnificación panelera y medios de vida ${ }^{1}$}

Acorde con lo anteriormente explicado, las figuras 2 y 3 muestran la influencia que ha tenido la tecnificación panelera en los capitales que conforman los Medios de Vida de los productores rurales, y la explicación se presenta por cada capital:

\footnotetext{
${ }^{1}$ Teniendo en cuenta la orientación cualitativa del estudio, se reconoce el uso del pentágono de bienestar, como herramienta que permite ilustrar mediante gráficos para la comprensión de los Medios de Vida de los productores paneleros. Es importante aclarar que en investigaciones de este corte, tanto resultados como discusión se pueden presentar de una forma conjunta con el fin de exponer su componente holístico.
} 


\section{VEREDA JUNCO ( PERIODO PREVIO A LOS AVANCES TECNOLÓGICOS)}

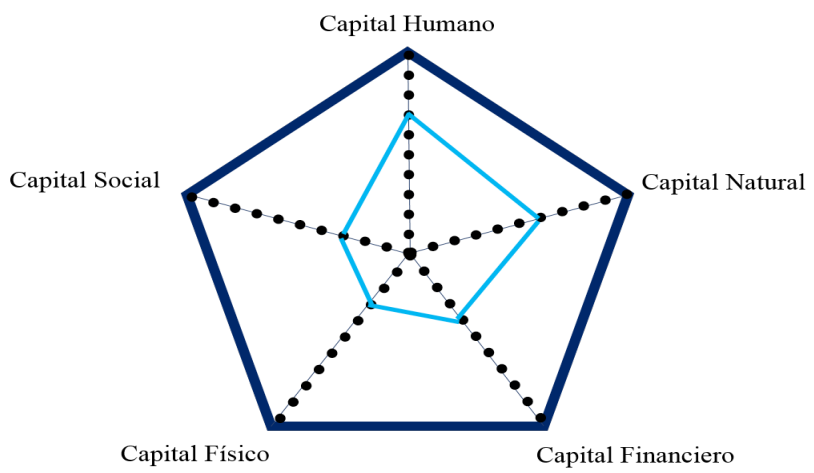

Figura 2. Pentágono del estado previo a los avances tecnológicos de los capitales

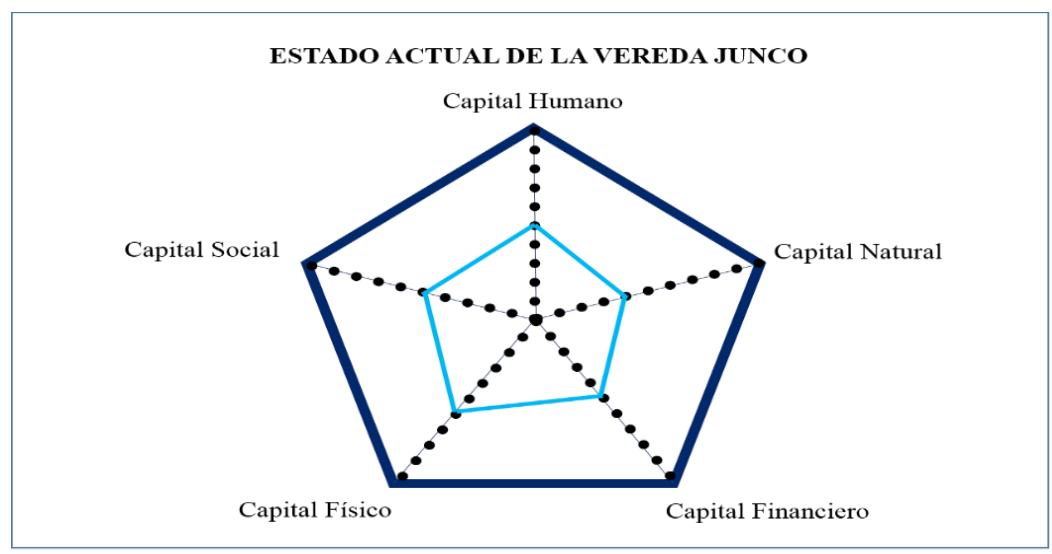

Figura 3. Pentágono del estado actual de los capitales 


\section{Capital Humano}

En la actualidad, el capital humano ha tenido una serie de cambios derivados de los distintos avances tecnológicos que han permeado la producción panelera; dichos cambios han influenciado más sobre algunas dimensiones en comparación con otras, es por esto que se muestran las principales características que han sido modificadas.

Los conocimientos actuales son el resultado de la hibridación entre la tradición popular y los aprendizajes recibo a través de la asistencia técnica, cabe resaltar que es mínima la presencia de instituciones formativas en el tema de producción panelera específicamente desarrolladas para el municipio, y que los procesos de capacitación solo han sido recibidos por pocos productores y proviene del Servicio Nacional de Aprendizaje - SENA.

En la dinámica de cambio que viene sufriendo la producción de panela, el campesino reconoce al SENA como principal actor y medio que le permite acceder a información sobre las regulaciones de la producción, nuevas tecnologías, Buenas Prácticas Agrícolas y Buenas Prácticas de Manufactura (SENA) (Ubaque, 2013, p.67).

Por otra parte, los productores que no han recibido asistencia técnica, consideran que es de suma importancia el hecho de aprender sobre las nuevas tecnologías, o las mejoras en el cultivo que han sido desarrolladas, y aclaran que esta debe ser provista por Estado.

En conocimiento lo que cada uno desarrolle, porque como no ha tenido la enseñanza la asistencia técnica, digamos que vengan y el expliquen al campesino así siempre, pero aquí ya es lo que cada uno ha desarrollado, como el campesino aquí nunca ha tenido el apoyo del gobierno, nunca han mandado una asistencia técnica que diga esto se siembra así, uno siembra los cultivos de maíz o un frutal lo siembra a su manera, como no sabe (PRO 14)2.

Una de las nuevas características frente al capital humano a nivel familiar, es el acceso a educación superior que están desarrollando algunos de los jóvenes, básicamente mujeres, hijas de productores paneleros bajo la modalidad de dueño de trapiche 'en esta época la gente es más estudiada, en esa época no había bachiller eso toco darles estudio, esas chinas', (PRO 3), y cuando ellas regresan a la región sus saberes sobre el proceso productivo son mínimos.

Un aporte importante en el capital humano, es el cambio de conocimientos como resultado de la nueva normatividad, que busca una producción más "limpia", de la panela 'entonces el gobierno cambió todas esas

\footnotetext{
${ }^{2}$ En adelante, los fragmentos de texto resaltados en letra tipo cursiva hacen referencia a relatos transcritos obtenidos mediante el trabajo de campo; estos relatos van acompañados de una codificación (PRO01), que se refiere al productor que suministró la información.
} 
leyes, la formó natural ahora es totalmente natural' (PRO 13), es decir dejando atrás el uso de químicos para su elaboración, que era parte de sus saberes tradicionales.

Dichos conocimientos, aportan al mismo tiempo a las habilidades que han sido potencializadas, en todo este proceso de avance tecnológico, es por esto que los productores actualmente son considerados más hábiles al momento de solucionar cualquier situación en la producción y el manejo sobre la maquinaria "la gente de ahora es pensaría uno que es como más inteligente, buscan las habilidades más fáciles, ponen como más habilidad a las cosas, sea se abren más espacios ponen a trabajar más máquinas" (PRO 13), lo cual claramente brinda algunas oportunidades para desarrollarse en su actividad productiva.

El relevo generacional en la producción de panela, se mantiene a través del tiempo en los conocimientos técnicos y productivos que son enseñados de una generación a otra. Esto trae consigo que la tradición permanezca presente en la población, pero que adopten los nuevos conocimientos, o desafíos de la actualidad a su saber popular (Ubaque, 2013).

Lo anterior indica que la aunque la región actualmente sigue siendo una de las principales productoras de panela en la Hoya del Río Suárez, lo que se estima es que en las próximas generaciones puede disminuir notablemente o incluso abandonarse esta actividad tradicional en la región, debido a la ausencia de interés y/o aportes de los más jóvenes en el cultivo de caña y las constantes migraciones por realizar estudios fuera del Municipio ('ya de poco de esa vaina, ellos les gusta montar en cicla y en moto' (PRO 3), 'no la verdad, pues no, por eso te digo que la juventud esta mentalizada en otras cosas que no es, o sea se mentalizan como en que cumplen cierta edad, bachillerato' (PRO 15)).

'Hay algunos no todos, pero hay algunos, por lo menos en las vacaciones se van hacer los oficios de la caña' (PRO 14). Además es importante resaltar, que algunos jóvenes, sobre todo aquellos que no cuentan con facilidades económicas para acceder a este tipo de educación superior, continúan desarrollando la producción panelera.

Puesto que este nuevo periodo ha traído algunos cambios en la producción, la mano de obra también se ha visto afectada, desde el uso de fungicidas para los cultivos, lo cual reduce el número de obreros "ha sido como los venenos sirven para una cosa porque no hay obra de mano que se facilite porque en un día pueden fumigar una hectárea de tierra dos obreros, mientras que en un día, varias semanas 10 obreros que no da el presupuesto" (PRO 4), hasta el gran aumento en la producción, que requiere mayor uso de obreros en las funciones propias del trapiche, debido a que la caña en las moliendas es muchísimo mayor. 
Así mismo, las condiciones de los obreros han cambiado, puesto que el número del personal disponible para las funciones de molienda ha disminuido, y se tiene el control sobre la producción, es por esto que los pagos han aumentado y las horas de trabajo han disminuido, 'hoy, entran a las cinco de la mañana y salen a las dos de la tarde ya no trabajan mucho' (PRO 8), lo que afecta directamente el proceso de elaboración de la panela, ya que todas sus funciones son cíclicas e indispensables.

\section{Capital Social}

Los productores paneleros manifiestan que actualmente no cuentan con un apoyo institucional sólido por parte de organizaciones tanto de carácter público y privado, ya que no reciben ningún tipo de capacitación y/u orientación sobre el cuidado de los cultivos, la actividad productiva, etc.; además no cuentan con ningún apoyo económico por parte del Estado, en términos de subsidios, por lo cual deben acceder a préstamos a entidades bancarias.

Se puede inferir que la red más cercana a los productores de la Vereda Junco, es el banco, específicamente el Banco Agrario,- información suministrada por el productor 9 en la realización del Diagrama de Venn, ya que estos les proporcionan intereses accesibles, ante la solicitud de préstamos de dinero.

En este sentido le corresponde a la administración municipal regular y ordenar la actividad de las unidades económicas, para consolidar su desarrollo económico autónomo y su inserción dominante a nivel subregional, logrando generar mayores beneficios e impactos económicos positivos en los habitantes del municipio (Alcaldía Municipal de San Benito, 2012).

La escasa asistencia estatal y del gobierno local en el desarrollo de la producción panelera, basándose en la narración de los productores, se refleja en que no hay una red de apoyo que provenga del gobierno Nacional, Departamental y Local. Ante ello, los productores expresan la pertinencia e importancia de recibir un apoyo institucional, basado en capacitaciones frente a las formas de cuidar el cultivo, uso de insumos, herramientas y maquinaria requeridos para la producción.

De modo similar, ocurre con la Federación Nacional de Panela (FEDEPANELA), entidad gremial que representa al sector panelero del país, quienes tienen la responsabilidad de recaudar dinero para invertir en planes y proyectos que beneficien al sub-sector panelero en campañas educativas, capacitaciones, asistencia técnica, entre otras (FEDEPANELA, 2016), ya que no están prestando dichos servicios establecidos en sus parámetros internos como institución, en la región de San Benito, de acuerdo con lo relatado por los productores. 
| Luisa Fernanda Cárdenas Franco, Natalia Espinosa Becerra, Olga Viviana González Pulido,

Hidali Carina Guasca Parra

FEDEPANELA si nos tienen más abandonados. Solo el nombre pero ese FEDEPANELA no hay apoyo de nada, no tiene diferente, digamos a los cafeteros que digamos tienen la cooperación de cafeteros si hay subsidios si hay prestamos FEDEPANELA no hay nada, una entidad para mí, es una entidad obsoleta (PRO16)

Frente a las redes comunitarias, se identifica que al igual que en años anteriores, los productores deben seguir acudiendo al alquiler de trapiches para sus moliendas, lo cual se conoce como maquilas, ya que muchos de ellos no cuentan con los medios económicos para la compra de estos "Moler en el trapiche del vecino, Lo que le toca uno mismo trabajar, o si no se tienen nada' (PRO 1). Esto permite identificar que en la Vereda Junco se han construido relaciones comunitarias basadas en la reciprocidad y ayuda mutua entre vecinos, en términos de empleo, préstamo de infraestructura para la producción, mano de obra, y recursos naturales.

Siguiendo el mismo orden, se evidencia el desconocimiento de algunos productores frente a la existencia de asociaciones de paneleros, en el sector "(...) No ahorita no hay organizaciones, No aquí cada quien lucha por lo suyo" (PRO 6), pero es importante señalar la presencia de dos asociaciones en la región, entendidas como organizaciones sin fines de lucro que colocan en un mercado común su producción individual, a partir de la agrupación de productores que realizan operaciones que no serían viables a nivel de productor individual, lo cual les permite visibilizarse en el mercado comercial (Rodríguez et al., 2004)

La primera es la Asociación de Campesinos de San Benito (ASOCASB), frente a ella, el presidente de la asociación comento el tiempo de su funcionamiento, el número de personas que la conforman, que acciones se realizan, los sucesos más importantes en su desarrollo y las repercusiones en la actualidad. Y la segunda, corresponde a la Asociación de productores de guayaba y cítricos de San Benito (APROGUCISB).

Sí, yo pertenezco a una asociación, eso está manejado tres líneas, se maneja productores de cítricos, productores de panela y productores de guayaba los que tenga guayaba pertenece a los de cítrico, los que tienen panela también pertenece, es lo que tenga mandarina también, Las mismas personas estamos en crisis qué vamos a hacer vamos a organizarnos entonces usted ya dice para ser una asociación que debemos tener entonces uno ya pide apoyo al SENA (PRO16)

Por otra parte, las relaciones familiares actuales dentro del proceso de producción panelera, conservan la tendencia de años anteriores, frente a las labores de los hombres como cortadores, alzadores, etc., mientras que 
las mujeres realizan acciones relacionadas con preparación y distribución de los alimentos a los obreros, aunque cabe resaltar que algunas mujeres, en especial, las propietarias de trapiches, están asumiendo un rol administrativo, ya que asumen el pago de jornales, la supervisión del proceso productivo, entre otras.

Yo soy la administradora, yo administro el personal, los obreros, la que me encargo del mercado, la que me encargo de pagarles, la que me encargo de todo y mi marido, mirar si el motor está bueno, la maquinaria y el equipo y el molino (PRO4)

\section{Capital Natural}

Los recursos naturales utilizados en la producción de panela, al igual que los periodos anteriores, aun continua siendo los más relevantes, el uso de la tierra y el agua, se puede afirmar, que en consecuencia del periodo de avances tecnológicos, estos dichos recursos han tenido una serie de transformaciones. Según Stocking y Murnaghan (2003) "muchos 'Medios de Vida' rurales depende del medio ambiente natural, por eso cualquier disminución permanente de la productividad de ese medio ambiente tendrá efectos adversos en las capacidades de las unidades familiares y las comunidades de mantenerse por ellas mismas" (p.33)

Además de lo expuesto, la modernización agroindustrial en los últimos años ha llevado consigo una serie de cambios en cada uno de los recursos naturales con los que cuenta una población para subsistir, entendiendo que el fenómeno de la tecnificación se venía desarrollando años anteriores y que se emplean medios tecnológicos en la agricultura. A continuación se realiza una descripción de las principales transformaciones que han tenido los recursos naturales de los productores rurales de la vereda Junco a partir de la tecnificación panelera, en cuanto al uso de la tierra y el agua.

Los cultivos de caña en los últimos años han invadido grandes extensiones de tierra con respecto a épocas anteriores y a otros cultivos, debido que la tradición de la región ha sido cultivar caña de azúcar para producir panela, esta ha sido relevada generacionalmente, y las nuevas generaciones adoptaron estas prácticas, y por esta razón aumento el cultivo de caña en la región.

En los últimos años la tendencia central ha sido la expansión de cultivos de caña en áreas que no había aprovechamiento de la tierra, dicho cultivo reemplazó otros cultivos aunque en algunas de las fincas, la siembra de caña hoy en día ocupa extensiones de tierra más grandes con respecto a los otros usos que se les da a las parcelas, y en otras, los terrenos donde había caña fue reemplazada por construcción de casa y por el cultivo de café. 
Asimismo, los animales utilizados en la fuerza de trabajo y por efectos del período de avances tecnológicos, han sido reemplazados por el uso de maquinarias, aunque algunos productores aun cuentan con mulas, bueyes y caballos para transportar la caña; estos han disminuido con relación a los animales de compañía, al igual que las épocas anteriores estos animales son de gran importancia en los terrenos de los productores rurales, porque tienen la tarea de cuidar: las casas, los trapiches y los cultivos; del mismo modo continua siendo la compañía de sus dueños; en cuanto a las gallinas y los pollos siguen siendo el plato principal en los alimentos. La permanencia de todos estos animales se encuentra alrededor de las fincas o por los caminos.

Actualmente, las semillas han sido reemplazadas por semillas certificadas por el Instituto Colombiano Agropecuario - ICA que proporcionan la Unidad Municipal de Asistencia Técnica Agropecuaria - UMATA con la finalidad que las plantas de caña crezcan de una forma más rápida con respecto a las semillas de tradición que son naturales, aunque esta práctica no ha sido acogida por la mayoría de los productores, ellos aún continúan con las semillas naturales, no obstante las semillas certificadas han presentado inconvenientes respecto de las semillas de tradición, porque al iniciar al iniciar la tecnificación en los métodos de siembra, hubo una afectación en el uso de los suelos, las cañas perdieron su fuerza y la resistencia que caracterizaban las antiguas. 'Las mejores cañas eran las antiguas, ahora las que salen no son iguales, las cañas antiguas eran más resistentes al clima y esa vaina. Se precisa más la tierra del cultivo ahora son más frágiles' (PRO 6)

El uso del agua se utiliza en mayor cantidad en la siembra, en el proceso de la panela y en el consumo humano 'Pues para el proceso del panela si se va bastante cantidad de agua pero en mi casa aquí la finca se ha sostenido con el agüita' (PRO 4) en las fincas aún permanece los aljibes pero estas han disminuido su capacidad de almacenamiento, con respecto a años anteriores, cabe resaltar que las normas han establecido prácticas agrícolas para la conservación del recurso hídrico, las cuales han sido adoptadas por los algunos de los productores y dueños de trapiche

El agua en la producción de panela, resulta un recurso valioso para los pobladores de la región, puesto que esta es utilizada en los cultivos, en el momento de la siembra, en el riego y para limpiarla, también en el proceso de la panela, además el agua es utilizada para limpiar los residuos que quedan después de las moliendas, pero la falta de lluvia y la contaminación a la que ha sido expuestas, por falta de prácticas adecuadas de los productores, ha contribuido en la disminución del recurso hídrico. 


\section{Capital Físico}

Con la llegada de los avances tecnológicos en la producción panelera se evidenciaron cambios significativos en los recursos físicos. Según Bedoya, Cárdenas y López (2015) “este capital está constituido por la infraestructura de carácter pública o privada y el conjunto de bienes y servicios con los que cuentan las personas para satisfacer sus necesidades básicas, realizar actividades productivas y alcanzar su desarrollo y bienestar" (p.41), estos cambios son a partir de las innovaciones propias de la globalización, y de las nuevas normas colombianas, que establecieron como requisito, el uso de nuevas tecnologías en la agroindustria, lo cual tuvo que ser adoptada por los productores paneleros.

El uso del azadón y el machete, herramientas que se usaban para retirar la maleza de la caña, en la actualidad son usadas en la siembra y en el momento del apronte. Aproximadamente hace 30 años, se reemplazó la práctica de proteger la caña de una manera tradicional con el uso de abonos de la tierra del café, la ceniza de la hornilla del trapiche entre otros, estos se aplicaban de manera manual, actualmente con la llegada de los fertilizantes, el uso de estas sustancias ha tenido un desarrollo significativo con la implementación de estacionarias eléctricas.

En los últimos años, se tecnificó en métodos de siembra, no obstante se utilizan las mismas herramientas, se adoptaron nuevas maquinarias para reducir el tiempo al momento de la siembra, a diferencia de años anteriores, el campesino duraba días haciendo las zanjas o en los arados. Hoy en día, y de manera rápida con la nueva maquinaria todas estas tareas no le llevan mucho tiempo al productor rural

Pues ahorita por lo menos antes la gente, por lo menos ahorita ya no se utiliza el azadón ya es el tractor bueno por lo menos se sigue utilizando el machete por lo menos ya antes la gente muchas veces llevaba la caña en las mulas hoy por lo menos ya la llevan en tractor o llevan en volqueta, pues la gente... pues se ha hecho un cambio técnicamente pero no es tan avanzado digamos más bien ha sido lento (PRO 16)

La región ha tenido un avance significativo en materia de vías, aunque todavía la carretera principal que inicia desde Güepsa y pasa por el área rural de San Benito hasta llegar a la zona urbana continúa en trochas (Alcaldía Municipal de San Benito, 2012), esto ha dado cabida a una mayor movilización de automóviles, desde hace unos años se puede acceder a las fincas por caminos que se limitaron, recordando que anteriormente se accedía por los barrancos, hoy en día en algunas de las fincas, los carros llegan cerca de las casas 'Ahora si está en buen estado' (PRO4) 
No obstante, cabe resaltar que la infraestructura vial, es un recurso físico valioso para los productores paneleros, para la comercialización de la panela a otras regiones, aunque estas no se han modernizado completamente a medida de la tecnología; o la normatividad no estableció vías adecuadas para un buen desarrollo en la agroindustria panelera, sin la carretera los productores de la vereda Junco no podrían vender las cajas de panela, y es importante mencionar que hace unos meses la alcaldía de San Benito inició con la pavimentación de algunos trayectos de la carretera, probablemente en la administración actual o en las próximas administraciones se termine de pavimentar toda la carretera 'es aquí, los alcaldes es por proyectos, sacan un proyecto de dos o tres kilómetros, y el otro tiene que sacar otro proyecto' (PRO 6).

En cuanto a la infraestructura, algunas construcciones de las casas y los trapiches en estos momentos aún continúan en obra gris, y otras construcciones ya están terminadas. En la actualidad los trapiches esta cubiertos por teja de Eternit ${ }^{\circ}$, a medida del paso de los años las construcciones fueron mejorando. Al inicio del nuevo milenio, los trapiches han experimentado cambios significativos en su modernización, en la actualidad para aumentar la producción se empezó adquirir motor con electricidad y motores Diesel, estos son más potentes y muelen mayor cantidad de caña.

\section{Capital Financiero}

El capital financiero ha sido uno de los activos que permitióla adopción de estos avances tecnológicos, ya que preveía el recurso económico para su adquisición, y desarrollo de cada uno de los momentos necesarios en la producción panelera. Los créditos han aumentado, es decir actualmente los productores obtienen mayores préstamos para diversas inversiones, pero aún sigue presente el destinado a la caña, en el caso del productor dueño de trapiche, una institución importante es el Banco Agrario. Por otra parte, los demás créditos solicitados dependen de las necesidades y capacidad de pago de cada productor.

Desde la perspectiva del productor panelero el aumento de los salarios a los obreros es significativo, debido a que hay más trabajo, menos obreros y la variación en el precio de la panela; también se debe tener en cuenta la alimentación que ellos reciben, donde los productores se refieren al alza de los precios en general, lo que requiere un mayor gasto de dinero por parte de los productores

Los productores paneleros bajo la modalidad de dueños de trapiche intentan ahorrar parte del dinero que han obtenido de las maquilas, esto cuando se tiene la posibilidad de hacerlo, 'Por ahí nosotros allá en el molino, les arrienda uno y vamos sacando la maquila' (PRO 5). Dichos ahorros se 
mantienen como forma de sostenibilidad para las diversas necesidades que se presenten, 'ahorros sí, pocos o muchos pero se ahorra, así corriente, para todo lo que se necesite' (PRO 6).

Las inversiones también han aumentado en la manera en que, los nuevos parámetros así lo exigen, esto se puede ver reflejado como una situación cotidiana, por la que atraviesa cualquier productor panelero en el país. Sin embargo, los medios para el logro de la producción que se exige, se convierten en un obstáculo para la adaptación a la nueva realidad productiva, a pesar, de reconocer la importancia de los cambios, para el productor, es difícil aceptar el nivel de las exigencias y los costos que significa adoptarlas (Ubaque, 2013, p.66).

Es por esto que se han realizado inversiones en el arreglo y adecuación del trapiche 'eso tocó invertir en el trapiche, que la hornilla, siempre hubo sus gastos, que toco cambiar la preparación del motor' (PRO 3). 'un trapiche no se va a arreglar con 20 millones eso toca como unos 300 millones para meterle todo lo que ellos dicen' (PRO 5), lo que reafirma grandes inversiones de capital para cumplir con la normatividad exigida, que se basa en la salubridad del trapiche.

Esto indica que las inversiones fueron representativas en dicho periodo de adecuación del trapiche, por otra parte en estos momentos, dichos intereses han cambiado debido a que los productores no buscan hacer inversiones a largo plazo en caña, como consecuencia a la inestabilidad de su precio, razón por cual proyectan invertir en nuevos cultivos.

Mientras exista exceso de oferta habrá unidades que se podrán ofrecer a un precio más bajo y mientras haya exceso de demanda habrá unidades que se comprarían a un precio más alto. Si el proceso de ajuste se deja funcionar bajo las libres fuerzas de mercado bajarán los precios cuando hay exceso de oferta y subirán cuando hay exceso de demanda (Velasco, 2011, p. 91).

En la actualidad las ganancias han disminuido debido a la poca estabilidad del precio de la panela, que ha sido fuertemente influenciado por la sobreoferta en el mercado, además de los nuevos egresos producto de créditos para inversiones, y de los gastos en el aumento de la mano de obra, y el valor de los jornales.

\section{Conclusiones}

El capital natural disminuyó debido a la escasez de agua y el mal estado en que se encuentra la caña, esto a consecuencia que algunos productores rurales no tienen conocimiento de prácticas agrícolas adecuadas para el 
uso de las nuevas herramientas que permitan la protección del agua y el cultivo; en relación a los animales, estos fueron reemplazados por las nuevas maquinarias y por los motores eléctricos. Cabe resaltar que el cultivo de caña aumento de manera gradual en toda la vereda, y esta representa una gran cantidad de extensión de tierra, y los animales aún continúa estando en el proceso del apronte.

El capital físico aumentó debido a que se empezaron a utilizar nuevas herramientas para la siembra y en el proceso, las vías se encuentra en mejor estado, los caminos están limitados para el acceso a las fincas, la infraestructura de los trapiches y las casas fueron mejorando, el acceso a los motores eléctricos han sido adquiridos por una parte de la población, y la capacidad de estos ha permito que sean arrendados a los cañicultores.

El capital humano ha disminuido en el relevo generacional debido a los constantes cambios de intereses de los jóvenes de la familia, sumado a la creciente migración de lo rural a lo urbano, por otra parte sigue presentándose en un nivel promedio ya que se han adquirido nuevos conocimientos frente a la técnica del cultivo y la producción de la panela, lo que hace que su ponderación se conserve.

Frente al capital social, se evidencia un ascenso en términos de la ponderación con relación al anterior, debido a la extensión del cultivo de caña, y por ende a la producción panelera, los campesinos de la Vereda Junco se han visto en la necesidad de asociarse, con el fin de ser más competitivos ante las demandas del mercado, y de esta manera poder ser visibles ante diversas entidades en el sector.

El estado del capital financiero aumentó, porque se presentan nuevos créditos y hay un mayor flujo de dinero entre la población, esto como resultado del aumento de los salarios, y el alza de los precios a nivel general, como también la proyección en inversiones en distintos cultivos, aunque hay que resaltar que las ganancias derivadas de la panela han disminuido por la fluctuación de los precios en el mercado y la sobreoferta.

El enfoque de los Medios de Vida Sostenibles permitió desarrollar una descripción en cada uno de los capitales con el fin de comprender las formas que la comunidad de la vereda junco de San Benito Santander desarrollaron estrategias en la sostenibilidad de cada uno de los capitales, de igual manera el presente artículo narra los cambios que ha tenido cada uno de ellos, lo cual visibiliza un diagnóstico de la población con respecto al fenómeno de la tecnificación panelera con el fin de crear nuevas estrategias encaminadas a la potencialización de cada uno de ellos. 


\section{Referencias bibliográficas}

Alcaldía Municipal de San Benito (2012). Plan de Desarrollo Municipio de San Benito 2012-1015. República de Colombia. Departamento de Santander, p. 55-60. Recuperado de http:// sanbenito-santander.gov.co/apc-aafiles/32303634313364363833643466633635/pdm-sanbenito-ok.-segunda-parte.pdf

Bedoya, G., Cárdenas, G. y López, M. (2015). Estrategias de Adaptación y Medios de Vida de las Familias Integrantes de la Fundación Consejo Veredal -Fcv-, Municipio De Calarcá, Quindío. Revista Luna Azul, (41) 201-239. Recuperado de http://www.redalyc.org/articulo. oa?id=321739268012

Bisquerra, A. (coord.) (2009). Metodología de la investigación educativa. Editorial La Muralla: Madrid. Recuperado de https://books.google.com.co/books?id=VSb4_cVukkcC\&pg=P A149\&dq $=$ se+localiza+algunos+individuos,+ los + cuales + conducen $+\mathrm{a}+\mathrm{otros}+,+\mathrm{y}+\mathrm{estos}+$

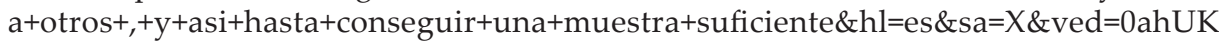
Ewj50t7t45XMAhUEdR4KHYCfDuQQ6AEIHzAB\#v=onepage\&q=se $\% 201$ localiza $\% 20$ algunos $\% 20$ individuos $\% 20 \% 2$ Clos $\% 20$ cuales $\% 20$ conducen $\% 20$ a $\% 20$ otros $\% 20 \% 2 \mathrm{C} \% 20$ y $\% 20$ estos $\% 20$ a $\% 20$ otros $\% 20 \% 2$ C $\% 20$ y $\% 20$ asi $\% 20$ hasta $\% 20$ conseguir $\% 20$ una $\% 20$ muestra\%20suficiente\&f $=$ false

Crespo, C. y Salamanca, A. (2007). El muestreo en la investigación cualitativa. Nure Investigación, (27), p. 2 - 4. Recuperado de http://www.sc.ehu.es/plwlumuj/ebalECTS/praktikak/ muestreo.pdf

Department for International Development DFID. (1999). Hojas Orientativas Sobre los Medios de Vida Sostenibles. Recuperado de http://community.eldis.org/.59c21877/SP-GS1.pdf

Federación Nacional de Productores de Panela FEDEPANELA (2016). ¿Quiénes somos? Recuperado de http://www.fedepanela.org.co/index.php/nuestra-federacion/quienessomos

García, D. (2015) Proyecto de prefactibilidad para un plan de negocios en la diversificación de productos de la panela en una finca productora de caña de azúcar. Trabajo de Grado, Especialización en Evaluación y Desarrollo de Proyectos. Universidad del Rosario. , Colombia. Recuperado de http://repository.urosario.edu.co/bitstream/handle/10336/11393/PLAN\%20DE\%20 NEGOCIO\%20DIVERSIFICACI\%C3\%93N\%20PANELA.pdf?sequence=1\&isAllowed=y

García, J. (2004). Estudios descriptivos. Nure Investigación. No. 7. Recuperado de http://www. fuden.es/ficheros_administrador/f_metodologica/formacion\%207.pdf

Geilfus, F. (2002). 80 herramientas para el desarrollo participativo: diagnóstico, planificación, monitoreo, evaluación. Instituto Interamericano de Cooperación para la Agricultura. Costa Rica.

Gottret, V. (2011) Una estrategia para el diseño e implementación de iniciativas para la reducción de la pobreza. El enfoque de los medios de vida sostenibles. Centro Agronómico Tropical de Investigación y Enseñanza (CATIE): Costa Rica. Recuperado de http://biblioteca.catie. ac.cr/descargas/Gottret_et_al_Guia_Enfoque_MdVS_Mod_I.pdf

Hernández Sampieri, R.; Fernández, C. y Baptista, P. (2010). Metodología de la investigación. McGraw-Hill / Interamericana Editores, S.A. de C.V. $5^{\text {ta }}$ edición. México.

Osorio, G. (2007) Manual técnico. Buenas prácticas agrícolas -BPA- y Buenas Prácticas de Manufactura -BPM en la producción de caña y panela. Corpoica, Convenio FAO-MANA. Recuperado de http://www.fao.org.co/manualpanela.pdf

Rada, G. (2007). Estudios descriptivos: Tipología. Universidad Católica de Chile. Recuperado dehttp://escuela.med.puc.cl/recursos/recepidem/PDF/epiDesc4.pdf

Rodríguez, G. (1998). La agroindustria panelera colombiana: análisis de la producción, distribución y consumos. Memorias del Congreso: Primera capacitación a nivel regional sobre filanejo 
| Luisa Fernanda Cárdenas Franco, Natalia Espinosa Becerra, Olga Viviana González Pulido, Hidali Carina Guasca Parra

y postcosecha del cultivo de caña panelera. p. 31-49. Caquetá. Colombia. Recuperado de http://bibliotecadigital.agronet.gov.co/jspui/bitstream/11348/4726/2/Memorias\%20 primera\%20capacitaci\%C3\%B3n.pdf

Rodríguez, G., García, H., Roa, Z. y Santacoloma, P. (2004). Producción de panela como estrategia de diversificación en la generación de ingresos en áreas rurales de América Latina. Servicio de Gestión, Comercialización y Finanzas Agrícolas (AGSF) Dirección de Sistemas de Apoyo a la Agricultura. FAO: Roma (Italia). Recuperado de http://www.fao.org/fileadmin/user_ upload/ags/publications/AGSF_WD6s.pdf

Salamanca, A. (2008). Estudios de motivación y percepción del consumidor de hortalizas orgánicas en la ciudad de Bogotá estratos 4,5 y 6 en el año 2009 (Trabajo de grado para optar por el título de Administrador de Empresas). Pontificia Universidad Javeriana. Bogotá (Colombia). Recuperado de http://www.javeriana.edu.co/biblos/tesis/economia/tesis74.pdf

Stocking, M. y Murnaghan, N. (2003) Manual para la evaluación de campo de la degradación de la Tierra. Recuperado de https://books.google.com.co/books?id=ADUTrX6Rx0kC\&pg=PA3 $3 \& l p g=P A 33 \& d q=$ capital + natural + en $+e l+$ campo\&source $=$ bl\&ots $=0 P K n H T k P j w \& s i g=0 \mathrm{~V}$ mmvL-Menb-CdgRx9phKFXIVXg\&hl=es\&sa=X\&ved=0ahUKEwic4NS1jdzLAhXJpR4K HReFBnYQ6AEIJjAC\# $\mathrm{v}=$ onepage\& $\mathrm{q}=$ capital $\% 20$ natural $\% 20 \mathrm{en} \% 20 \mathrm{el} \% 20$ campo\&f $\mathrm{f}=\mathrm{false}$

Superintendencia de Industria y Comercio (2012). Cadena productiva de la panela: Diagnóstico de libre competencia. Gobierno de Colombia. Recuperado de http://www.sic.gov.co/ recursos_user/documentos/promocion_competencia/Estudios_Economicos/Panela2012. pdf

Ubaque, L. (2013). Gestión en la Producción Panelera, Municipio de Villeta, Cundinamarca. Trabajo de grado, Maestría en Gestión Ambiental. Pontificia Universidad Javeriana. Bogotá, Colombia. Recuperado de http://repository.javeriana.edu.co/bitstream/10554/12385/1/ UbaqueGonzalezLuzLilia2013.pdf

Velasco, O. (2011). Equilibrio y fuerzas de mercado. Universidad Nacional de Plata. Argentina. Recuperado de http://sedici.unlp.edu.ar/bitstream/handle/10915/27080/04EQUILIBRO+Y+FUERZAS+DE+MERCADO.pdf?sequence=1 